\title{
Atherosclerosis prediction by microarray-based DNA methylation analysis
}

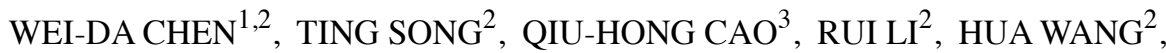 \\ ${\mathrm{XIU}-\mathrm{BAO} \mathrm{CHEN}^{2} \text { and ZE-TAO CHEN }}^{2}$ \\ ${ }^{1}$ Health Care Department, Shandong University of Traditional Chinese Medicine, Jinan, Shandong 250355; \\ ${ }^{2}$ Health Care Department, Affiliated Hospital of Shandong University of Traditional Chinese Medicine, \\ Jinan, Shandong 250011; ${ }^{3}$ Department of Anesthesiology, Jinan Center Hospital, \\ Jinan, Shandong 250013, P.R. China
}

Received July 4, 2018; Accepted April 29, 2020

DOI: $10.3892 /$ etm.2020.9025

\begin{abstract}
Using a series of DNA methylation analysis, pathogenesis was investigated to identify the specific DNA methylation markers for diagnosing atherosclerosis. Firstly, with the chip platform of Illumina Human Methylation 450 BeadChip, a total of 1,458 CpGs, covering 971 differential methylated genes were extracted with stringent filtering criteria. Secondly, hierarchical clustering as a heat map was used to check on the dependability of differential methylated genes. Thirdly, the related GO terms and pathways were enriched by up- and down-methylated genes, respectively, after verifying the capacity of these differential methylated genes to distinguish between atherosclerosis and healthy controls. In total, 971 differential DNA methylated genes were identified (1,458 CpGs). Several important function regions were also identified, including cell adhesion, PI3K-Akt signaling pathway and transcription from RNA polymerase II promoter. This study indicates that patients with atherosclerosis have high levels of DNA methylation, which is promising for early diagnosis and treatment of atherosclerosis.
\end{abstract}

\section{Introduction}

Atherosclerosis is a multifactorial and chronic disease, which is generally hallmarked by chronic inflammation, lipid storage, endothelial dysfunction and vascular calcification in the vasculature (1-3). This disease will lead to series of severe consequence, such as heart attack, stroke and gangrene of the extremities (4). Many therapies have been used for this disease, including nicotinic acids, fibrate derivatives, statins, bile acid sequestrants and cholesterol absorption inhibitors (5). Although

Correspondence to: Dr Ze-Tao Chen, Health Care Department, Affiliated Hospital of Shandong University of Traditional Chinese Medicine, 42 Wenhuaxi Road, Jinan, Shandong 250011, P.R. China E-mail: chenzetaobio@126.com

Key words: atherosclerosis, DNA methylation, GO terms, pathways contemporary intervention and pharmacological agents have been applied to this disease, because of the irreversible damage on tissues and organs, the treatment on the atherosclerosis is not ideal. Therefore, it is imperative to find effective biomarkers for early discovery and to carry out preventive treatment.

Many scholars have focused on the relationships between DNA methylation and atherosclerosis. DNA methylation is the most studied epigenetic modification in human beings $(6,7)$. Simultaneously, a great number of human diseases, such as cancer and Parkinson disease $(8,9)$ have been confirmed to be related to aberrant DNA methylation. As the important regulator of gene transcription, DNA methylation is a heritable and stable epigenetic marker (10). Therefore, confronting the chronic disease with no specific therapeutics, research on DNA methylation could be recognized as a novel direction for early treatment and diagnosis of this disease.

In the present study, after analyzing the 15 atherosclerosis samples and 15 normal controls downloaded from the ArrayExpress database, the differential DNA methylated genes were extracted from the total samples. In order to verify the capacity of these genes in differentiating atherosclerosis and normal controls, clustering analysis was performed on these genes. Subsequently, analysis of these differential methylated genes were applied to the GO and pathway analysis. Finally, the related genes were mapped to the relevant GO terms and biological pathways. Through these significant GO terms and pathways, it was anticipated that the related functions with atherosclerosis could be found to elucidate the pathogenesis and identify molecular makers for early diagnosing and treatment of the disease.

\section{Materials and methods}

DNA methylation data recruiting and preprocessing. The genome-wide DNA methylation aberrations (E-GEOD46394) in human atheroscleosis were downloaded from the ArrayExpress database (http://www.ebi.ac.uk/arrayexpress/) and were deposited on the A-AFFY-44 - Affymetrix GeneChip Human Genome U133 Plus 2.0 [HG-U133_Plus_2]. The E-GEOD-46394 was comprised of 30 samples, including 15 atherosclerosis samples and 15 normal controls. The age 
range of the samples is from 45 to 88 years on the basis of the common age of this disease. Simultaneously, the sex of the atherosclerosis samples and normal controls were randomly selected, all containing men and women. The clinical characteristics of these samples are shown in supplement Table SI. From the database, a total of $485,577 \mathrm{CpGs}$ in the DNA methylation data were obtained by us. However, three types of probe should be removed, including the singlenucleotide polymorphism (SNP) with the distance from $\mathrm{CpG}$ to $\mathrm{SNP} \leq 2$, the probe with minor allele frequency (MAF) $\leq 0.05$ and the probe on cross-hybridising and sex chromosome. A total of 427,909 methylation data with $\mathrm{CpGs}$ were obtained for the following analysis after the preprocessing.

Screening of differential methylated genes. The value of the methylation expression profiles was defined as the $\beta$-values with range between 0 (no methylation) to 1 (complete methylation) (11). In order to identify the differential methylated CpGs, two stringent criteria were strictly observed. The absolute $\beta$-values of the difference between atherosclerosis and the healthy groups had to be $>0.5$, and that the P-value should be $<0.05$ after t-test. Through the preliminary screening, a total of 58,903 differential methylated CpGs were extracted including 42,932 up-methylated CpGs and 15,971 down-methylated CpGs (representing 13,245 genes).

In order to ensure that the data could be used to facilitate subsequent analysis, further filtering steps should be performed for the differential methylated genes. Two conditions had to apply. First step is that the differential methylated $\mathrm{CpGs}$ with $\beta$-value $\leq 0.2$ or $\geq 0.8$ in all samples should be deleted. The average absolute $\beta$-values of difference between atherosclerosis and the healthy groups should be $\geq 0.2$. Finally, the differential methylated genes were the simplified data, which could be used for the following studies.

Clustering analysis. Clustering analysis is a Pattern Recognition techniques $(12,13)$. In order to establish that the differential methylated genes extracted from the database could distinguish atherosclerosis groups from the normal controls, clustering analysis should be used to verify the effectiveness. In the present study, the effective data visualization was presented on the heatmap by Pheatmap package in $\mathrm{R}$ language 3.4 version, setting the conditional parameter as the absolute percentage methylation values ( $\beta$-values) of the difference between the atherosclerosis and the healthy groups as $>0.5$. The other criterion was that the P-value should be $<0.05$ after t-test, which represent a dataset with two dimensions, commonly genes and samples (14). Ultimately, through this heatmap, the distribution of the level of the differential methylated $\mathrm{CpGs}$ in all samples were clearly found.

$G O$ analysis. Due to the vast differential methylated data generated from the microarray experiments, GoMiner's query gene ontology (GO) database can be used to assess the function of differentially expressed genes (15). The GO database (http:/www.geneontology.org/) is an open source tool that covers three domains, which is widely used in functional annotation and enrichment analysis $(16,17)$. Through the GO analysis, it is expected to discover the important function related to the methylation changes and to find the differential methylated genes corresponding to the related function regions. In the present study, setting the threshold value of $\mathrm{P}=0.01$, we discarded the differential methylated genes with poor P-value and carried out the enrichment on the rest of genes with the category of biological process. Ultimately, the related differential methylated genes were mapped to the same GO terms, which could be used to check the function regions.

Remarkably, the differential methylated genes mentioned above could be divided into two types of genes that, respectively, were up-methylated and down-methylated. However, the biological function of the two types of genes was the opposite. When genes are transcribed into mRNA, the positively regulated up-methylated genes will promote gene expression and the negatively regulated, down-methylated genes will inhibit gene expression. In consequence, in order to find out the respective biological function of the two types of genes, the GO analysis was applied respectively.

Pathway analysis. The difference is that the two methods focus on different approaches and tools employed for the task. Generally speaking, pathway is a process that proteins interacted with each other to regulate cell function and metabolic activity. As is know, the occurrence of a disease in biological pathway arises from the expression change not only in the individual gene but also in the interactions between related genes. Pathway analysis can help in discovery of the most important biological pathway correlated to the disease. In this study, the Kyoto Encyclopedia of Genes and Genomes (KEGG) (http://www.geneme.jp/kegg/) was the common pathway database (18). Using the stringent filtering criteria of the pathways enriched by the differential methylated genes with $\mathrm{P}<0.05$, the related pathways were extracted. Similarly, two types of pathways were presented, respectively, enriched with the up-methylated and down-methylated genes.

\section{Results}

Identification of differential methylated genes between atherosclerosis samples and normal controls. Analyzing the 15 atherosclerosis samples and 15 normal controls with the chip platform on Illumina Human Methlation 450 BeadChip, 427,909 methylated CpGs were obtained. Subsequently, for the sake of the following analysis, there were two procedures to filtrate the differential methylated genes. The first is the preliminary screening with the criteria of the absolute $\beta$-values of the difference between the atherosclerosis and the healthy groups $>0.5$ and the $\mathrm{P}<0.05$. Ultimately, a total of 58,903 differential methylated CpGs including 42,932 up-methylated CpGs and 15,971 down-methylated CpGs were obtained. Details of these genes are presented in Fig. 1. Subsequently, further filtering steps should be applied to facilitate a more stringent analysis. The criterion that was applied discarded the CpGs with $\beta$-values $\leq 0.2$ or $\geq 0.8$ in all samples and the retained $\mathrm{CpGs}$ with the absolute $\beta$-values were the difference between the atherosclerosis and the healthy groups of $>0.2$. Ultimately, 1,458 differently methylated CpGs covering 971 genes extracted by us were considered as the final results.

Hierarchical clustering analysis on the differential methylated genes. The purpose of extracting the differential 


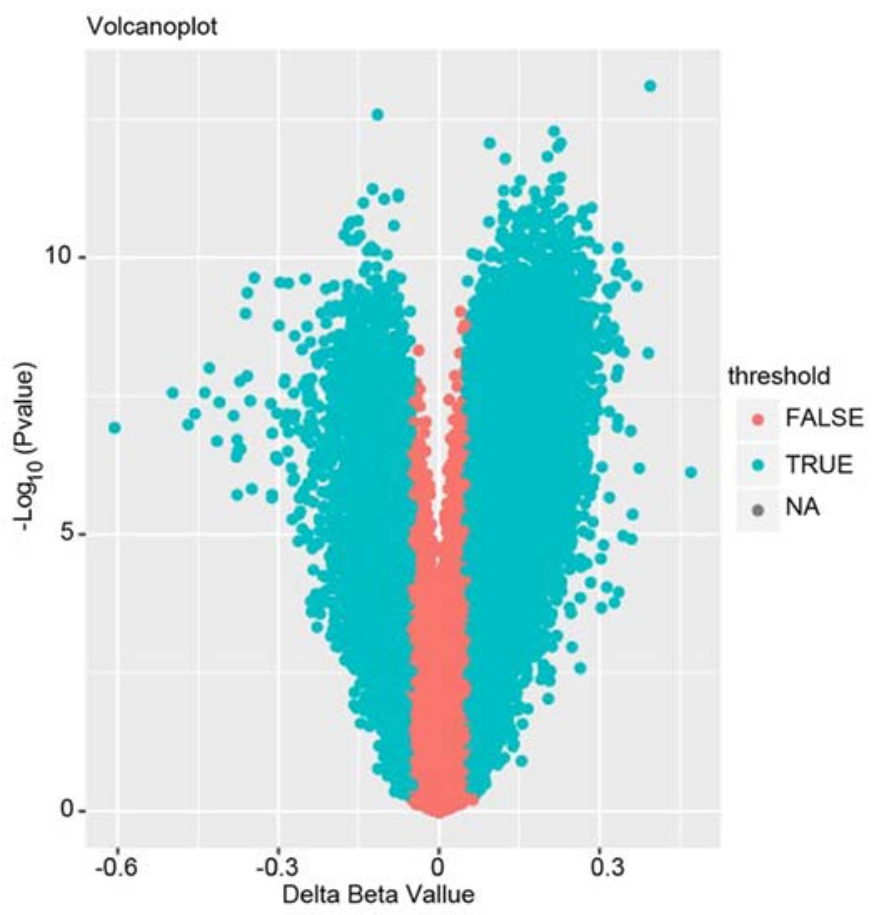

Figure 1. Volcano plot of the up-methylated and down-methylated genes. Figure shows mean methylation differences between atherosclerosis and control (x-axis) versus $\log$ transformed P-values (y-axis). Blue points on the left represent the true down-methylated genes and the others on the right represent the true up-methylated genes.

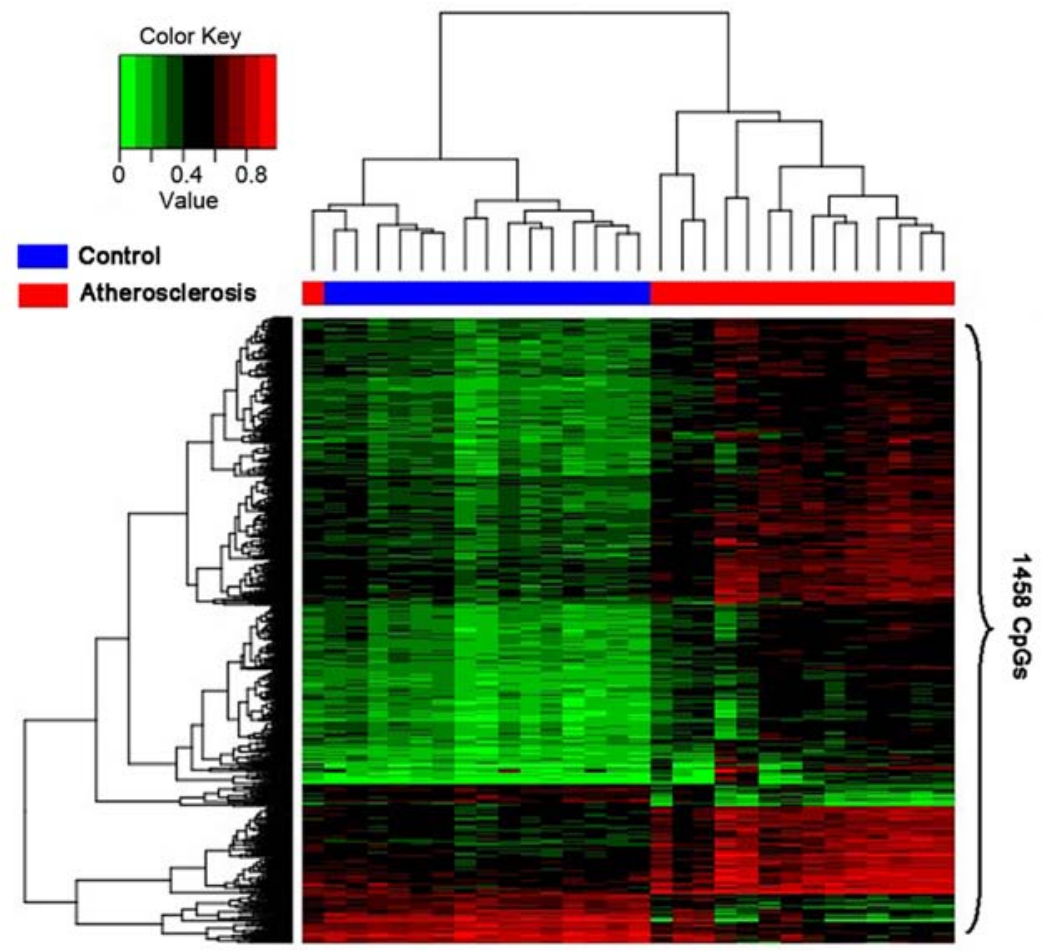

Figure 2. Hierarchical clustering was performed as a heatmap, which shows distribution of the differential CpG methylation level and the high and low methylation in samples. The column and row, respectively, represent the samples and the $\mathrm{CpG}$ sites. The color shows a quantitative measure of methylation for each CpG site with range between 0 (no methylation) to 1 (complete methylation). Green represents the low methylation and red represents the high methylation. Healthy controls and atherosclerosis samples are shown by the blue and red bars, respectively.

methylated genes was to confirm the related GO terms or biological pathways based on these genes, which could help us to make certain the mechanism of the occurrence of atherosclerosis and discover the approach to diagnose 
Table I. Last 30 GO terms with P-value enriched with the up-methyalted genes.

\begin{tabular}{|c|c|c|}
\hline GO terms & P-value & FDR \\
\hline Cell adhesion & 4.47E-07 & 2.83E-07 \\
\hline Positive regulation of GTPase activity & $2.24 \mathrm{E}-05$ & $1.40 \mathrm{E}-05$ \\
\hline Anterior/posterior pattern specification & 4.37E-05 & $2.75 \mathrm{E}-05$ \\
\hline Positive regulation of bone mineralization & $1.12 \mathrm{E}-04$ & $6.94 \mathrm{E}-05$ \\
\hline Positive regulation of endothelial cell migration & $1.52 \mathrm{E}-04$ & $9.43 \mathrm{E}-05$ \\
\hline Negative regulation of osteoblast differentiation & $2.50 \mathrm{E}-04$ & $1.56 \mathrm{E}-04$ \\
\hline Glossopharyngeal nerve morphogenesis & $3.30 \mathrm{E}-04$ & $2.06 \mathrm{E}-04$ \\
\hline Positive regulation of transcription, DNA-templated & $3.81 \mathrm{E}-04$ & 2.39E-04 \\
\hline Peptidyl-tyrosine phosphorylation & $4.21 \mathrm{E}-04$ & 2.64E-04 \\
\hline Thymus development & $5.04 \mathrm{E}-04$ & $3.15 \mathrm{E}-04$ \\
\hline Positive regulation of phosphatidylinositol 3-kinase signaling organization & $5.17 \mathrm{E}-04$ & $3.24 \mathrm{E}-04$ \\
\hline Transforming growth factor beta receptor signaling pathway & $7.00 \mathrm{E}-04$ & 4.37E-04 \\
\hline Regulation of epithelial cell migration activity & 7.97E-04 & 4.97E-04 \\
\hline Negative regulation of transcription from RNA polymerase II promoter & $9.97 \mathrm{E}-04$ & $6.22 \mathrm{E}-04$ \\
\hline Cartilage development & 0.001039 & $6.51 \mathrm{E}-04$ \\
\hline Cellular response to transforming growth factor beta stimulus & 0.001243 & 7.87E-04 \\
\hline Positive regulation of catenin import into nucleus & 0.001394 & $8.66 \mathrm{E}-04$ \\
\hline Embryonic skeletal system development & 0.00172 & 0.001081 \\
\hline Central nervous system development & 0.002427 & 0.001510 \\
\hline Extracellular matrix organization & 0.002647 & 0.001651 \\
\hline Negative regulation of transcription, DNA-templated & 0.002654 & 0.001654 \\
\hline Positive regulation of epithelial to mesenchymal transition & 0.002868 & 0.001791 \\
\hline Stress fiber assembly & 0.00346 & 0.002158 \\
\hline Negative regulation of sodium ion transmembrane transporter activity & 0.004041 & 0.002519 \\
\hline Lymph vessel development & 0.004041 & 0.002519 \\
\hline Positive regulation of MAP kinase activity & 0.004157 & 0.002599 \\
\hline Integrin-mediated signaling pathway & 0.004187 & 0.002618 \\
\hline Nervous system development & 0.004236 & 0.002652 \\
\hline Ephrin receptor signaling pathway & 0.004477 & 0.002786 \\
\hline Axon guidance & 0.004509 & 0.002815 \\
\hline
\end{tabular}

FDR, false discovery rate.

Table II. GO terms with P-value enriched with the down-methylated genes.

\begin{tabular}{lll}
\hline GO terms & P-value & FDR \\
\hline Anterior/posterior pattern specification & $7.46 \mathrm{E}-06$ & $4.68 \mathrm{E}-06$ \\
Transcription from RNA polymerase II promoter & $3.18 \mathrm{E}-04$ & $1.99 \mathrm{E}-04$ \\
Positive regulation of transcription from RNA polymerase II promoter & 0.001554 & 0.000974 \\
Negative regulation of transcription from RNA polymerase II promoter & 0.002829 & 0.001774 \\
Cell development & 0.008129 & 0.005063 \\
Thymus development & 0.00935 & 0.005842 \\
Regulation of vasculogenesis & 0.010149 & 0.006375
\end{tabular}

FDR, false discovery rate.

or treat the disease. Therefore, it is imperative that the reliability of the differential methylated genes should be verified. The results of the analysis will be displayed by the heatmap. In consequence, the heatmap (Fig. 2) was able to make clear that the disease samples had been separated from the controls. 
Table III. The 30 pathways with P-value enriched with the up-methylated genes.

\begin{tabular}{ll}
\hline Pathways & P-value \\
\hline Focal adhesion & $3.60 \mathrm{E}-05$ \\
PI3K-Akt signaling pathway & $2.72 \mathrm{E}-04$ \\
Ras signaling pathway & $9.84 \mathrm{E}-04$ \\
Regulation of actin cytoskeleton & 0.001012 \\
HIF-1 signaling pathway & 0.003851 \\
Glutamatergic synapse & 0.0044 \\
Rap1 signaling pathway & 0.005313 \\
cAMP signaling pathway & 0.006573 \\
Pathways in cancer & 0.008167 \\
Cholinergic synapse & 0.009791 \\
Endocytosis & 0.010149 \\
Axon guidance & 0.010285 \\
Mucin type O-Glycan biosynthesis & 0.01085 \\
Adrenergic signaling in cardiomyocytes & 0.012417 \\
Adherens junction & 0.012676 \\
ErbB signaling pathway & 0.014367 \\
ECM-receptor interaction & 0.014367 \\
Proteoglycans in cancer & 0.015585 \\
Hippo signaling pathway & 0.016108 \\
Tight junction & 0.017989 \\
Fatty acid metabolism & 0.018393 \\
Osteoclast differentiation & 0.030227 \\
TGF-beta signaling pathway & 0.031804 \\
cGMP-PKG signaling pathway & 0.032235 \\
Insulin secretion & 0.033815 \\
Choline metabolism in cancer & 0.034316 \\
Prostate cancer & 0.040362 \\
Fatty acid degradation & 0.043614 \\
Oxytocin signaling pathway & 0.047152 \\
Morphine addiction & 0.047703 \\
\hline & \\
\hline
\end{tabular}

GO analysis on the differential methylated genes. The purpose of performing GO analysis on the differential methylated genes is to establish the important function related to the methylation changes and find the differential methylated genes corresponding to the related function regions. By setting the threshold value at $\mathrm{P}=0.01$ and the threshold value at $\mathrm{FDR}=0.01$, the differential up and down-methylated genes, respectively, enriched the GO terms according to the classification of biological process. The classification results are presented in Tables I and II, which clearly illustrate the relevant function enrichment.

Pathway analysis on the differential methylated genes. The pathway analysis was performed with the up-methylated and down-methylated genes independently, which could be expected to discover the related function corresponding to the development of this disease. In consequence, the results are presented in Table III. However, different from the GO analysis there were no down-methylated genes mapped to the related biological pathways and only 50 pathways presented with up-methylated genes. For easy observation, only those in the last 30 pathways with P-value enriched with the differential methylated genes were chosen, as they were considered the most relevant biological pathways.

\section{Discussion}

As a chronic inflammatory disease, atherosclerosis is involved in activation of adaptive and innate immunity (19). Hypertension, smoking and diabetes could all become the incentives for this disease (20). The pathogenesis of the disease has been widely researched by many scholars, including formation of aggregating homocysteinylated lipoproteins with microorganisms (21), reactive oxygen species (22), and microRNA-155 (23). However, the pathogenesis has not been fully elucidated yet. Moreover, because of the irreversible damage on tissues and organs, the treatment on atherosclerosis is not ideal. Therefore, in order to clarify the occurrence and development and reduce the incidence of this disease, finding the biomarkers for the early detection and prevention has become critical in dealing with the occurrence of atherosclerosis. Epigenetics is emerging as an attractive candidate for the update and future therapy perspectives in atherosclerosis (24). Epigenetics may not only help understanding the molecular mechanisms of atherosclerosis as genetic predisposition explains only part of cardiovascular disease risk, but also play an important role in early diagnosis of some diseases, such as cancer and adult-onset disease (25). Therefore, as the most studied epigenetic modification, DNA methylation was investigated in atherosclerosis by us.

In the present study, 971 differential DNA methylated genes (1,458 CpGs), including up-methylated and down-methylated genes were identified with the stringent filtering criteria. Then, after enriching the up-methylated and down-methylated genes into the GO terms and biological pathways respectively, we sought out several critical GO terms and pathways with lower P-value, which could be considered as the important function to reveal the occurrence and development of this disease.

Through the enrichment analysis with up-methylated genes, there were GO terms and some pathways that attracted our attention, including the GO terms with cell adhesion and the pathway with Focal adhesion, PI3K-Akt signaling pathway, Ras signaling pathway and HIF-1 signaling pathway. Previously, there has been considerable research focused on the relationship between cell adhesion and atherosclerosis. Additionally, there have been research on the treatment of atherosclerosis on mice indicating that bilirubin can prevent atherosclerotic plaque formation by inhibiting monocyte migration through the disruption of endothelial vascular cell adhesion (26). This discovery demonstrated that the cell adhesion played an important part in the development of the atherosclerosis. The other point that deserves attention is the PI3K-Akt signaling pathway. Similarly, this pathway has been focused on by many scholars. Among them, Zhai et al (27) found that the selective inhibition of PI3K-Akt signaling pathway would markedly affect atherosclerotic plaque inflammation. In addition, by experiments on mice, quercetin and myricitrin were confirmed to inhibit atherosclerosis via PI3K-Akt signaling pathway (28). Research on the cell adhesion and PI3K-Akt signaling pathway confirmed that it can reveal the development of this disease.

Literature has documented that targeting Ras homolog gene family member A (RhoA) inhibits the proliferation 
and migration of vascular smooth muscle cells (VSMCs) identified as major cellular events in hypertension-induced vascular remodeling, closely involved in the progression of atherosclerosis, implicating targeting Ras signaling is a promising strategy for treating atherosclerosis $(29,30)$. In addition, previous studies have demonstrated that HIF-1, as one of the main regulators of cellular responses in a low-oxygen environment, is recognized to have vital roles in the development of atherosclerosis via cell-specific responses, reacting on endothelial cells, VSMCs and macrophages and emphasized to behave as a potential therapeutic target on the atherosclerosis development $(31,32)$. Feng et al (33) concluded that HIF1 $\alpha$ triggers atherosclerosis initiation by inducing excessive endothelial cell proliferation and inflammation via inducing production of glycolysis enzymes. Altogether, these enrichment pathways hold regulatory implications for atherosclerosis initiation and development, which provides some valuable insight or feasible means for treating atherosclerosis in further clinical studies.

Furthermore, the GO terms and pathways enriched by the down-methylated genes deserve attention. After careful analysis, the GO term transcription from RNA polymerase II promoter with the relatively low P-value became a focus of our attention, which may have great relevance with the occurrence of atherosclerosis. Specifically, human cytomegalovirus (HCMV) has been thought to be the most possible etiological factor of atherosclerosis (34). During late HCMV infection with atherosclerosis, this virus facilitates viral transcription by regulating the elongation rate of RNA polymerase II (35). Therefore, it can explain the close relationship between RNA polymerase II and the development of this disease.

Factors such as the location and degree of methylation (that is, a specific residue can bind to different numbers of methyl groups) can influence whether gene expression is promoted or inhibited. Uncovering the underlying mechanism of gene methylation affecting targeted genes is of significance for our work and further study.

Increasing evidence has identified that DNA methylation is involved in multiple processes and diseases, including atherosclerosis (36-38). As shown in the supplement Table SII of up-methylated genes, namely, HOXA3, PIK3R2, FOXD3, MAP3K8, ITPKB, IGF1R, and FOXC2. It is reported that methylation sites located in HOXA3 are identified to relate with cholesterol efflux capacity (39). In addition, Balakrishnan et al (40) demonstrated that promoter DNA methylation status of PIK3R2, FOXD3, MAP3K8, ITPKB, IGF1R, and FOXC2 were validated by bisulfite DNA sequencing involved in activation of insulin signaling and angiogenesis. Furthermore, as presented in supplement Table SIII of downmethylated genes, namely, HOXD4, SMAD3, HDAC4. It is determined that HOXD4 and HOXA2 are found to be most pronouncedly hypomethylated in carotid atherosclerotic plaques in comparison with their methylation patterns in intact tissues of interior mammary arteries and saphenous veins (41). HOXD4 is also confirmed to be hypomethylated in the independent dataset of the right coronary arteries in advanced atherosclerotic plaques compared with the other tissues of vascularture (42). Riffo-Campos et al (43) identified that SMAD3 is recognized to be a hub regulator correlated with metal-related differentially methylated region and atherosclerosis; moreover, HDAC4 is discerned to be associated with effectors of atherosclerosis.
In conclusion, compared with the traditional method, the study of atherosclerosis with DNA methylation used related GO terms and pathways related to atherosclerosis. After the analysis, the GO terms and pathways termed cell adhesion, PI3K-Akt signaling pathway and transcription from RNA polymerase II promoter were considered to be the most related function regions with the development of the disease. As a discoverybased study, our findings may provide a potential biomarker for the early detection and prevention. Although the analysis results require clinical data of large samples as support, it still provides a new research direction for studying atherosclerosis.

Some limitations are present in the current work. Data from bioinformatics analysis was used in the whole study. Pure bioinformatics analysis is not prior to integrated bioinformatics and experimental verification, which is a limitation for our present study. This work focused on uncovering GO function and pathways involved in aberrant DNA methylation, thereinto a mass of methylated genes were included. It is difficult to realize a small range of gene level detection or methylation level or status detection, using RT-PCR, MSP and Bisulfite sequencing. Additional tools for pathway analysis are required. Reactome and IPA were used to perform pathway analysis and found less pathways were enriched.

In the present study, we preliminarily chose the KEGG, as a common pathway enrichment analysis method. Further research is necessary, with more datasets or our clinical sample collection, combined with more systematic analytical means.

\section{Acknowledgements}

Not applicable.

\section{Funding}

No funding was received.

\section{Availability of data and materials}

The datasets used and/or analyzed during the current study are available from the corresponding author on reasonable request.

\section{Authors' contributions}

WC and TS conceived the study and drafted the manuscript. $\mathrm{RL}$ and $\mathrm{HW}$ acquired the data. XC and ZC analyzed the data and revised the manuscript. All authors read and approved the final version of the manuscript.

\section{Ethics approval and consent to participate}

Not applicable.

\section{Patient consent for publication}

Not applicable.

\section{Competing interests}

The authors declare that they have no competing interests. 


\section{References}

1. Hasanov Z, Ruckdeschel T, Kapel S, Mogler C, Appak S, Spegg C and Augustin HG: Role of Endosialin during atherosclerosis progression. Atherosclerosis 241: e14, 2015.

2. Salic K, Wielinga PY, Verschuren L, Gjorstrup P, Kleemann R and Kooistra T: Intervention with anti-inflammatory RVE1 attenuates atherosclerosis without decreasing plasma cholesterol and adds to the anti-atherogenic effect of atorvastatin. Atherosclerosis 235: e267, 2014.

3. Campbell LA and Rosenfeld ME: Infection and atherosclerosis development. Arch Med Res 46: 339-350, 2015.

4. Ross R: The pathogenesis of atherosclerosis: A perspective for the 1990s. Nature 362: 801-809, 1993.

5. Campbell JH, Efendy JL, Smith NJ and Campbell GR: Molecular basis by which garlic suppresses atherosclerosis. J Nutr 131: 1006-1009, 2001.

6. Portales-Casamar E, Lussier AA, Jones MJ, MacIsaac JL, EdgarRD, Mah SM, Barhdadi A,Provost S, Lemieux-Perreault LP, Cynader MS, et al: DNA methylation signature of human fetal alcohol spectrum disorder. Epigenetics Chromatin 9: 25, 2016.

7. Razin A and Riggs AD: DNA methylation and gene function. Science 210: 604-610, 1980.

8. Esteller M and Herman JG: Cancer as an epigenetic disease: DNA methylation and chromatin alterations in human tumours. J Pathol 196: 1-7, 2002.

9. Masliah E, Dumaop W, Galasko D and Desplats P: Distinctive patterns of DNA methylation associated with Parkinson disease: Identification of concordant epigenetic changes in brain and peripheral blood leukocytes. Epigenetics 8: 1030-1038, 2013.

10. Gopalakrishnan S, Van Emburgh BO and Robertson KD: DNA methylation in development and human disease. Mutat Res 647: $30-38,2008$

11. Wu P, Farrell WE, Haworth KE, Emes RD, Kitchen MO, Glossop JR, Hanna FW and Fryer AA: Maternal genome-wide DNA methylation profiling in gestational diabetes shows distinctive disease-associated changes relative to matched healthy pregnancies. Epigenetics 13: 122-128, 2018.

12. Chen CY and Ye F: Particle swarm optimization algorithm and its application to clustering analysis. In: IEEE International Conference on Networking, Sensing and Control. IEEE, Taiwan pp789-794, 2004.

13. Diday E and Simon JC: Clustering analysis. Commun Cybern 10: 47-94, 1976.

14. Deu-Pons J, Schroeder MP and Lopez-Bigas N: Heatmap: An interactive heatmap viewer for the web. Bioinformatics 30 1757-1758, 2014.

15. Kestler HA, Müller A, Kraus JM, Buchholz M, Gress TM, Liu H, Kane DW, Zeeberg BR and Weinstein JN: VennMaster: Area-proportional Euler diagrams for functional GO analysis of microarrays. BMC Bioinformatics 9: 67, 2008.

16. Ashburner M, Ball CA, Blake JA, Botstein D, Butler $\mathrm{H}$, Cherry JM, Davis AP, Dolinski K, Dwight SS, Eppig JT, et al: Gene ontology: tool for the unification of biology. The Gene Ontology Consortium. Nat Genet 25: 25-29, 2000.

17. Du Z, Zhou X, Ling Y, Zhang Z and Su Z: agriGO: A GO analysis toolkit for the agricultural community. Nucleic Acids Res 38: 64-70, 2010

18. Ogata H, Goto S, Sato K, Fujibucji W, Bono H and Kanehisa M: KEGG: Kyoto encyclopedia of genes and genomes. Nucleic Acids Res 27: 29-34, 1999.

19. Kim HJ: Role of nucleotide-binding and oligomerization domain 2 protein (NOD2) in the development of atherosclerosis. Korean J Physiol Pharmacol 19: 479-484, 2015.

20. Bernhard D, Pfister G, Huck CW, Kind M, Salvenmoser W, Bonn GK and Wick G: Disruption of vascular endothelial homeostasis by tobacco smoke: Impact on atherosclerosis. FASEB J 17: 2302-2304, 2003.

21. McCully KS: Homocysteine and the pathogenesis of atherosclerosis. Expert Rev Clin Pharmacol 8: 211-219, 2015.

22. Goncharov NV, Avdonin PV, Nadeev AD, Zharkikh IL and Jenkins RO: Reactive oxygen species in pathogenesis of atherosclerosis. Curr Pharm Des 21: 1134-1146, 2015.

23. Ma X, Ma C and Zheng X: MicroRNA-155 in the pathogenesis of atherosclerosis: A conflicting role? Heart Lung Circ 22: 811-818, 2013.

24. Khyzha N, Alizada A, Wilson MD and Fish JE: Epigenetics of atherosclerosis: Emerging mechanisms and methods. Trends Mol Med 23: 332-347, 2017.
25. Bustos FJ, Ampuero E, Jury N, Aguilar R, Falahi F, Toledo J, Ahumada J, Lata J, Cubillos P, Henríquez B, et al: Epigenetic editing of the Dlg4/PSD95 gene improves cognition in aged and Alzheimer's disease mice. Brain 140: 3252-3268, 2017.

26. Kawamoto R, Ninomiya D, Hasegawa Y, Kasai Y, Kusunoki T, Ohtsuka N, Kumagi T and Abe M: Mildly elevated serum bilirubin levels are negatively associated with carotid atherosclerosis among elderly persons. PLoS One 9: e114281, 2014.

27. Zhai C, Cheng J, Mujahid H, Wang H, Kong J, Yin Y, Li J, Zhang Y, Ji X and Chen W: Selective inhibition of PI3K/Akt/ mTOR signaling pathway regulates autophagy of macrophage and vulnerability of atherosclerotic plaque. PLoS One 9: e90563, 2014.

28. Lu XL, Zhao CH, Yao XL and Zhang H: Quercetin attenuates high fructose feeding-induced atherosclerosis by suppressing inflammation and apoptosis via ROS-regulated PI3K/AKT signaling pathway. Biomed Pharmacother 85: 658-671, 2017.

29. Cui C, Wang X, Shang XM, Li L, Ma Y, Zhao GY, Song YX, Geng XB, Zhao BQ, Tian MR, et al: lncRNA 430945 promotes the proliferation and migration of vascular smooth muscle cells via the ROR2/RhoA signaling pathway in atherosclerosis. Mol Med Rep 19: 4663-4672, 2019.

30. Yu MH, Lin MC, Huang CN, Chan KC and Wang CJ: Acarbose inhibits the proliferation and migration of vascular smooth muscle cells via targeting Ras signaling. Vascul Pharmacol 103-105: 8-15, 2018.

31. Jain T, Nikolopoulou EA, Xu Q and Qu A: Hypoxia inducible factor as a therapeutic target for atherosclerosis. Pharmacol Ther 183: 22-33, 2018

32. Tanaka T and Eckardt KU: HIF activation against CVD in CKD: Novel treatment opportunities. Semin Nephrol 38: 267-276, 2018.

33. Feng S, Bowden N, Fragiadaki M, Souilhol C, Hsiao S, Mahmoud M, Allen S, Pirri D, Ayllon BT, Akhtar S, et al: Mechanical activation of hypoxia-inducible factor $1 \alpha$ drives endothelial dysfunction at atheroprone sites. Arterioscler Thromb Vasc Biol 37: 2087-2101, 2017.

34. Chen R, Xiong S, Yang Y, Fu W, Wang Y and Ge J: The relationship between human cytomegalovirus infection and atherosclerosis development. Mol Cell Biochem 249: 91-96, 2003.

35. Perng YC, Campbell JA, Lenschow DJ and Yu D: Human cytomegalovirus pUL79 is an elongation factor of RNA polymerase II for viral gene transcription. PLoS Pathog 10: e1004350, 2014.

36. Tabaei S and Tabaee SS: DNA methylation abnormalities in atherosclerosis. Artif Cell Nanomed Biotechnol 47: 2031-2041, 2019.

37. Jiang D, Sun M, You L, Lu K, Gao L, Hu C, Wu S, Chang G, Tao $\mathrm{H}$ and Zhang D: DNA methylation and hydroxymethylation are associated with the degree of coronary atherosclerosis in elderly patients with coronary heart disease. Life Sci 224: 241-248, 2019.

38. Aavik E, Babu M and Ylä-Herttula S: DNA methylation processes in atheosclerotic plaque. Atherosclerosis 281: 168-179, 2019.

39. Sayols-Baixeras S, Hernáez A, Subirana I, Lluis-Ganella C, Muñoz D, Fitó M, Marrugat J and Elosua R: DNA methylation and high-density lipoprotein functionality - brief report: The REGICOR study (Registre Gironi del Cor). Arterioscler Thromb Vasc Biol 37: 567-569, 2017.

40. Balakrishnan A, Guruprasad KP, Satyamoorthy K and Joshi MB: Interleukin-6 determines protein stabilization of DNA methyltransferases and alters DNA promoter methylation of genes associated with insulin signaling and angiogenesis. Lab Invest 98: 1143-1158, 2018

41. Nazarenko MS, Markov AV, Lebedev IN, Sleptsov AA, Frolov AV, Barbash OL and Puzyrev VP: DNA methylation profiling of the vascular tissues in the setting of atherosclerosis Mol Biol (Mosk) 47: 398-404, 2013 (In Russian).

42. Nazarenko MS, Markov AV, Lebedev IN, Freidin MB, Sleptcov AA, Koroleva IA, Frolov AV, Popov VA, Barbarash OL and Puzyrev VP: A comparison of genome-wide DNA methylation patterns between different vascular tissues from patients with coronary heart disease. PLoS One 10: e0122601, 2015.

43. Riffo-Campos AL, Fuentes-Trillo A, Tang WY, Soriano Z, De Marco G, Rentero-Garrido P, Adam-Felici V, LendinezTortajada V, Francesconi K and Goessler W: In silico epigenetics of metal exposure and subclinical atherosclerosis in middle aged men: Pilot results from the Aragon Workers Health Study. Philos Trans R Soc Lond B Biol Sci 373: 1748, 2018. 